\title{
Integration: Achieving Coherence Between Education and Practice in the Professions
}

\author{
Margaret McMillan, Penelope Little \\ School of Nursing and Midwifery, University of Newcastle, Australia
}

Integrated health services, focused on prevention, anticipate needs and support people to manage conditions, especially in compromised environments. Learning about contemporary, integrated health care is best achieved through integrated approaches to education. Contexts of care are crucial moderators of ways professionals respond to demands. The world of work is complex: In health services measures of safe and therapeutic practice differ across settings; cultures and contexts vary for different client groups; there is a range of capacities and levels of competence within professional and inter-professional groups; and, there are different approaches to managing well in crises. Implicit in successful, rapid responses to crises, are the capable workforces with particular abilities to deal with new situations. The authors suggest that Problem-based Learning (PBL) principles underpinning ideas on integration within and across education and practice, allow for validation of relevant concepts. Goals for Person-Centred Care (PCC) espoused by the World Health Organization apply in a range of situations, including the COVID19 scenario and will be reflected in students' learning outcomes. Given COVID19 challenges, health professions (within healthcare and education systems) recognized the need to adapt and innovate to provide effective patient care and ensure ongoing learning for existing and emerging professionals. Technologies assisted change processes, with limited resources. However, the extent of compromise on levels of integration in practice and education is unknown. Answers to questions posed here will assist with responses to global disruptive phenomena; lessons learned provide direction for management of chronic diseases, healthier living and engagement with the professional education that facilitates rapid responses to novel situations.

Keywords: Integration; Education for the professions; Frameworks for education and practice

\section{INTRODUCTION}

In this paper we examine the current dynamic environment that has arisen because of the pandemic. Responses to COVID 19 demand health professionals who are creative problem-solvers and work with others to deliver quality care appropriate to the context and the individuals. We argue that there is a priority for professionals who can respond to novel situations within an integrated framework. Educational methodologies like Problem-based Learning (PBL) lead to learning outcomes (abilities) that can be used in actual practice. In responding to this priority and the complexities arising from the COVID19 pandemic, the authors reinforce the idea that educating health professionals requires the use of a conceptual framework of professional education that aligns with that of integrated care. PBL curricula provide scaffolding for integration of ideas and facilitate modification of learning activities when necessary.

(C) Copyright 2020 International Society for Problem-Based Learning

(c) This is an Open Access article distributed under the terms of the Creative Commons Attribution Non-Commercial License (http://creativecommons.org/licenses/ by-nc/4.0/) which permits unrestricted non-commercial use, distribution, and reproduction in any medium, provided the original work is properly cited. 


\section{BACKGROUND}

The contexts of care and learning are crucial moderators of the way emerging and practicing professionals respond to demands on them. The world of work and ongoing professional development is complex: Measures of safe and therapeutic practice differ across settings; cultures and contexts vary for different client and student groups; there is a range of capacities and levels of competence within professional and inter-professional groups; and, there are different approaches to managing well within crises in a variety of health service and educational structures and processes.

For example, given the impact of the emergence of the COVID19 challenge (News, The Lancet, 2020), health professions (within the healthcare and education systems) were forced to adapt and innovate to continue to provide effective patient care and ensure ongoing learning for existing and emerging health professionals. E-technologies assisted the change processes, even where there were limited resources. However, the Deloitte Center for Higher Education Excellence (2020) highlighted the extent to which the pandemic has upended business as usual for colleges and universities. Not only have campuses shifted to remote learning almost overnight, but institutions are also suddenly grappling with grave financial challenges as the domestic and global economies may now face what looks to be a severe recession.

Aristovnik et al. (2010) completed a comprehensive and largescale study on student perceptions of the impacts of the COVID19 crisis on their lives. A study sample of 30,383 students from 62 countries, reported on perceptions on various aspects of the worldwide lockdown and transition to online learning. Support provided by teaching staff and their universities' public relations offices was a source of satisfaction for students. But limitations arising from their deficient computer skills and the perception of a higher workload 'prevented them from perceiving their improved performance in the new teaching environment'. Students have had to engage differently with educational programs.

The demand for a rapid shift in focus is now a global phenomenon; changes in health services will provide direction for the future if the professions are to adequately assist people to stay alive, manage their chronic diseases and live healthier lives at home.

Across the world, the response to the pandemic was well-publicized by the World Health Organization (WHO, 2020). In Australia, we have seen that an integrated health service and care system with a focus on prevention, anticipated needs and supported people to manage their conditions especially in compromised environments. Learning about contemporary, more integrated health care can best be achieved through an integrated approach to education. However, quality educational processes can be readily compromised in crisis situations.

WHO (2016) argued for an orientation towards more integrated care to meet aspirations for more Person-centered Care (PCC): In Australia, a rapid response to the COVID19 crisis was evident in the way health services and higher education were able to 'pivot' to work with structures and processes to respond rapidly to situations in a person-centered way. The WHO support for a more strategic 'all-encompassing' approach seems relevant in crisis management; the coordinators within service provision reflect their ambition to:

- Put people and their needs first

- Re-orient the model of care

- Re-organize the delivery of services

- Engage patients, their families and carers

- Rearrange accountability mechanisms

- Align incentives

- Develop human resources for health

- Uptake innovations

- Partner with other sectors and civil society

- Manage change strategically.

(WHO Regional Office for Europe. Lessons for transforming health services delivery: Compendium of initiatives in the WHO European Region).

\section{CONTEXT AS A DETERMINANT OF STRATEGY}

Professionals working in any field require access to evidence-based knowledge and know-how. In health services, the latest evidence informs care delivery processes in pursuit of optimal patient outcomes and greater assurance of quality and safety for both consumers and peers. We saw that evidence from experts on COVID19 informed decisions about how to achieve integration of services and processes in, and from a range of workplaces.

Blueprints for education, training and professional development should lead to relevant learning outcomes on which the clinician can build when novel situations present in the rapidly changing contexts. Professionals will rely on particular core abilities if they are to respond well to pressing issues, for example:

- Crises such as the emergence of COVID19

- Demographic changes and the impact of those trends on health, education and social policy and practice

- Less than optimal care and concern for people, instead of care as needed and in the right places

- Fiscal restraint and the drive for greater efficiencies. 
Identifying and developing the required core abilities of practitioners within health services is the challenge for education for and within the health professions. Hendry (2017) identified the core elements of integrated practice as those that center on

- context/care/service provision,

- person orientation,

- involvement in care processes,

- self-determination/choice and

- growth potential.

Education for integrated practice and models of integrated care are both informed by core concepts and values, such as those described by Hendry, and principles relevant to all aspects of health professional practice. Consumers are at the center of designs for service provision; students as consumers of education programs are central to learning in preparation for practice roles.

\section{IMPLEMENTATION OF INTEGRATED CARE}

To achieve outcomes espoused by Hendry (2017), the design and delivery of care needs to demonstrate

- Coordination and continuity of care

- Trusted relationships

- Accessible information and advice

- Consistent communication with, and between, staff

Hendry suggested there was a particular need for Registered Nurses (RNs) with specialist qualifications who have the abilities to work with GPs and healthcare support workers to

- promote self-management and independence

- use skilled assessment working with a person and their family to develop their care plan

- focus on prevention and anticipatory care management and coordination of care

- ensure collaboration with other disciplines

- use knowledge of local resources/networks.

Vulnerable populations are always the focus of policymakers. Older Australians, those impacted by mental distress and those with symptoms of a range of chronic diseases demanded special attention during the pandemic. Many had existing co-morbidities. Hendry (2017) from Scotland, provided evidence of a paradigm shift in approaches to the management of people with one or more symptoms of chronic diseases across the world. What matters to consumers Hendry said is that: "My care is planned with people who work together to understand me and my carer(s), put me in control, coordinate and delivery services to achieve my best outcomes" (National Voices, 2012) Health professionals, especially nurses who are accustomed to learning about and dealing with an older clientele, have been prominent in the societal response to COVID19; nurses were central to both practice redesign efforts in acute care and preventive strategies to ensure public safety. Hendry outlined what she described as 'anticipatory care interventions' that are targeted and tailored to the individual

- Self-management and advice and support including for dementia

- Polypharmacy reviews of safety, efficacy and adherence

- 'Thinking ahead', 'anticipatory' electronic care plans,

- Physical activity, falls prevention and management

- Identification and support for carers

- Coordinated case management for complex support

- Re-enablement and 'step-up/step-down' Intermediate Care

- Comprehensive geriatric assessment for frail older people

- Tele-health and tele-care

- Equipment for adaptations.

However, relationships and interactions need to be facilitative, indicative of partnerships, considerate of legal and human rights and the maintenance of dignity and respect for the recipient of care. Given that health and health-breakdown information is now more readily available online, health literacy is also encouraged, especially among the diverse cultural groups within the broader populations.

While there are specialized fields like cancer and mental health care which focus on working with consumers to meet their recovery and re-enablement goals, consideration of the person's physical, psychological, social and spiritual needs is also paramount. Today in most fields of health service, care processes need to consider the context of the person's lived experience, partnerships with their family, significant others and the broader community. Providing support for consumers and their families during life crises and transition periods is critical. Professionals in Australia have to liaise discretely and effectively with a range of health care providers, provide information and education on health maintenance and restoration, and coordinate care across contexts that span metropolitan, regional, rural and remote areas. The health workforce needs to be flexible and responsive and able to work with people across the life span, and in a variety of workplace settings, especially during crises.

As noted above, sustaining a more integrated approach to both practice and education demands a strategy informed by principles. These also apply to modifications made to blueprints for preparation for practice in all professional fields in uncertain circumstances such as that thrown up by COVID19. When the nature of work and workplaces change, different approaches to practice and education are required. In our local health services, 
one recent focus for change as a response to COVID19, related to managing people with chronic disease and multi-morbidities outside of the acute care environment. Primary health care came to the fore. Evidence had already suggested that those health systems involving primary health care were more efficient, have lower rates of hospitalization, fewer health inequalities and better health outcomes, including lower mortality (Australian Government, Department of Health, 2020). Health and information technologies have now opened up further access to all kinds of services, extended options on who can deliver services and where the service is delivered.

Before the emergence of COVID19, drivers for change in models of care and system redesign had already informed Australian Health Plans such as the NSW Health (2016) pilot studies for the implementation of more integrated care. Of particular interest was a focus on bi-directional physical and behavioral interventions for primary care clinicians. But also recognized as critical, was the need for assessment and management of mental health and substance abuse. However, additional considerations in responses to COVID19 included the importance of the creation of safe and healthy work environments, excellence in personalized patient care, meaningful education for patients and their carers and the wider population, leadership in innovation, health and information literacy, safe medication management, research and community services.

\section{EDUCATION FOR CHANGE}

As already noted, implicit in the successful contribution of the professionals' role in change is the development of the type of education programs that develop particular core abilities (applied learning outcomes) derived from an analysis of the professional practice, workplace and societal contexts. Working in inter-professional teams to deliver person-centered care has long been a goal of health services. Organizational structures have often facilitated the achievement of these outcomes. In a previous consultancy undertaken by one of the authors, staff members from the community, aged care and disability and educational services came together. These staff members were taken through a process to generate what they considered the core capabilities of all staff should be, provide parameters to guide inter-professional practice, drive organizational change, and maintain the collaborative, person-centered culture of the unit. The following core competencies (abilities) were identified through an analysis of data collected through workshops, focus groups, interviews, and practice narratives:

1. Provides person-focused care
2. Contributes to multi-professional client management;

3. Uses an evidence-based approach to practice

4. Engages in creative problem solving

5. Engages in ongoing professional development

6. Accepts shared responsibility for the Organizational Unit

The notion of shared responsibility was thought by all levels of staff participants to be the essence of collaborative practice and change and creative problem-solving at both the individual client and the broader systems levels; this was considered essential to the provision of optimal integrated care (Conway et al., 2011). While integrated care focuses on the individual consumer as central to the delivery of services, we acknowledge that the achievement of this outcome requires a whole of services approach and involves patients, clients, carers, family, volunteers, communities, institutions, and governing bodies.

Fostering the required capabilities in existing staff in health services through appropriate and relevant education and training can only be achieved when education and training take place in the context of workforce capability and organizational development. The approaches acknowledge systems within organizations or services that inhibit the application of the education and training outcomes and contribute to the development of a culture consistent with the elements of a 'learning organization'.

Educational methodologies and curricula designs such as Problem Based Learning (PBL) and Context-Based Learning $(\mathrm{CBL})$ have long espoused the centrality of professional practice in education for the professions. The rationale for, and underpinning philosophy of $\mathrm{PBL}$, argues that the world has been experiencing such rapid change that the goal of professional education is to equip graduates with the required abilities to respond to change and novel situations: Critical thinking, problem-solving and situation-analysis, self-directed learning and ongoing development, and an ability to work in intra- and inter-professional teams. PBL approaches achieve these outcomes by using a carefully selected range of situations from practice as the context for learning. Integration of knowledge from contributing disciplines is more meaningful for students. The use of appropriate performance-based assessment in PBL also results in the integration of knowledge, skills and values when responding to novel situations.

All university, vocational education and health service program development and implementation are subject to cyclical review processes guided by the higher and further education sectors. Participation of academics and clinicians from the practice arena provides a chance for an appreciation of maintenance of a level of integration in design and implementation processes. However, in the COVID19 situation, educational institutions were forced to move rapidly to more blended approaches reliant on e-learning. 
Some had systems in place; others needed to revolutionize more traditional ways. Hence decisions on organizational, practice and education renewal should also have included resources that support appropriate education and training.

The more blended and integrated approaches to education might have had some advantages in the crisis. A perusal of websites and program offerings suggests some providers have well developed conceptual frameworks for integration within program offerings; some espouse integrated philosophies and methodologies, and some directly relate to integrated services.

\section{DESIGNING CURRICULA FOR INTEGRATED CARE}

When articulating a pathway to integration, it is essential criteria and supporting values and principles form part of any overarching framework. When using a conceptual framework for a curriculum design that equips health professionals with abilities to participate in, provide and promote Integrated Care, it is necessary to align educational and practice conceptual frameworks. Over the years the International Foundation for Integrated Care (IFIC) has developed and used a series of building blocks as a conceptual framework that supports the successful delivery of integrated care services: There website outlines these as

- Shared Values and Vision

- Population health and local context

- People as partners in care

- Resilient communities and new alliances

- Workforce capacity and capability

- System-wide governance and leadership

- Digital Solutions

- Aligned payment systems

- Transparency of Progress, results and impacts.

An "examination of practice through curriculum development processes results in testing the congruence of existing conceptual frameworks of any discipline in the reality of contemporary practice". Scrutiny can raise questions about the relevance of espoused values and theories of professional practice to contemporary and future practice (Conway \& Little 2000). McMillan \& Little (2016) illustrated the congruence between models for professional activities and education with some examples of elements of both. Conway \& Little (2000) also argued that when PBL curricula reflects practice, staff members will articulate professional and/or discipline values and their educational practice values. Through the application of these to the 'real-life' situations captured in learning packages, the staff themselves repeatedly test their espoused theories of education and their profes- sion/discipline. They are required to demonstrate the process skills that underpin the PBL curriculum. This testing of assumptions, defining concepts of the profession/discipline and education and applying them in the context of their own 'real-life' teaching situations are the ongoing challenges for academics and clinicians in implementing PBL (Conway and Little, 2000).

\section{CORE CONTENT, STRUCTURE AND DELIVERY MECHANISMS}

The knowledge, skills and attitudes for greater integration in practice and education exist in health service plans and education provider policy frameworks that guide curriculum development, implementation and renewal. Integration of core concepts is necessary: Recovery/re-enablement, consumer-practitioner partnerships, therapeutic relationships, consumer-centered co-constructed care and ethical and other issues relevant to professional practices. When considering the curriculum structure and delivery mechanisms that best accommodate learning events which embrace concepts and content relevant to contemporary practice, modularization is a useful way to achieve the renewal of content when necessary. A significant component of any curriculum design is the assessment that provides evidence of learning outcomes (graduate abilities). Responses to COVID19 had the potential to undermine adherence to best practice principles for assessment tasks (in terms of graduate abilities) needed for any learning event: Equity; transparency of performance criteria; a balance of reliable and valid activities that support student learning. Workplace learning was impacted as workplaces changed, given the potential spread of infection. But as curriculum plans changed, in response to the pandemic, three principles still needed to inform the choice of assessment activities based on outcomes related to performance in the workplace.

\section{CONTEXTUAL RELEVANCE TO THE WORKPLACE}

Achieving the abilities defined in the learning outcomes requires the application of knowledge, skills and professional behaviors to workplace situations and demonstration of an ability to respond appropriately in these situations. The workplace situations used as stimulus material for learning should reflect the demands of the real world of practice and the potential for transformation. A range of assessment tasks employing application to a workplace context can collect evidence of this ability. 


\section{INTEGRATED, PERFORMANCE- BASED ASSESSMENT}

Assessment of workplace competence requires a broad range of performance-based evidence to support the achievement of the learning outcomes (graduate abilities). The focus of the assessment tasks should center on the integration of knowledge, skills and professional behaviors to develop appropriate responses to workplace situations.

\section{EVIDENCE-BASED ASSESSMENT}

The process of assessing learning outcomes expressed as graduate abilities requires the accumulation of evidence regarding individual performance over time and from a range of valid and reliable assessment modes. The assessment of performance should involve feedback from academics, clinicians, peers, self, consumers and other relevant stakeholders. Mapping processes will ensure appropriate coverage and weighting of assessments and links to each of the learning outcomes. Evidence gathered of achievement of learning outcomes (graduate abilities) should include practical demonstrations of achievement, including work environment demonstrations, simulation exercise, scenario or roleplay.

Indirect evidence will be available from work environment supervisor reports, workplace documentation, and written responses to problems/situations, scenarios and consumer-story vignettes.

Responses limited the usual choices from the range of examples of relevant assessment tasks to the pandemic: Written examination, assignment, consumer-based, solution-based, Objective Structured Clinical Examinations (OSCE), consumer-story vignettes study, or viva. Some options were no longer possible for safety reasons.

Despite the need for rapid responses to the crisis, the evidence of performance should still be obtained during modules, units, workshops, clinical practice to inform a judgment of competence and confidence. The evidence should readily align, through mapping, with the identified learning outcomes (graduate abilities).

\section{DISCUSSION}

Through the efforts of the IFIC strong theoretical foundations are emerging, the evidence-base has been tested and are underpinned by values which ensure that care processes and treatment are therapeutic, that is helpful and salient, especially during the COVID19 crisis (https://integratedcarefoundation.org/realis- ing-the-true-value-of-integrated-care-beyond-covid-19). While some core values are not unique to particular disciplines, they underpin the learning outcomes and the principles underpinning learning and teaching across the health professions.

During the COVID crisis, nurses had a distinct contribution in that there is a purposeful and time-intensive contact with the consumer, and commonly also with their families and significant others. This is important for people experiencing mental illness and distress during the pandemic. In our experience with education and policy forums, mental health care provides an excellent example of the importance of integration in health care and education processes. Nurses engage with the everyday lived experience of consumers and 'understand how this experience is complicit in their health profile and consequences'. The distinct way of relating and interrelating with consumers and their families distinguishes mental health professionals in their practice; these ideas were part of a framework for studies in that field (McMillan \& Little, 2016). However, mental health challenges and distress feature alongside a range of other symptoms of a disease; the same concepts can contribute to the development of a values-based conceptual framework for practices aspiring to greater integration in care processes and outcomes that are:

- Person-centered, in tune with the person's 'life needs' as well as their health needs; aspirations for recovery and re-enablement, are always central to care plans

- Reliant on the health professional's world view, i.e. individual commitment to consumer-generated care that is consistent with the best available evidence

- Centered on mutually beneficial collaboration with consumers and others through partnerships

- Consistent with opportunities to reflect on plans that meet peoples' needs.

In summary, the distinctive contribution of integrated care evident in health services is characterized by an approach that is collaborative and co-constructed with the consumer and their family. As a consequence, there is a commitment to re-enablement and recovery for individuals receiving health care. The philosophy and understandings about the nature of the practice should be readily evident in the conceptual models of educational preparation for beginning and more experienced professionals. It should reflect current research into optimal practices and outcomes. McMillan and Little (2016), when developing a Framework for post-graduate studies in mental health nursing in Australia, argued that benchmarks guide best practice in developing, implementing and maintaining curriculum integrity. Values and principles were consistent with aspirations for integrated practice and education. Practices are driven by policies that drive integra- 
tion in and across practice and curriculum documents (subject/ unit outlines, assessments, clinical placement records and schedules) student evaluations, formal agreements with clinical agencies and involvement of health service consumers and family/ carer representatives.

However, the IFIC membership, in their advertisements for forthcoming forums, suggest that many questions remain if we are to achieve an appropriate level of integration in health services and care. These involve

The Future of Health and Care - What are the challenges, opportunities and unknowns that need to be considered by health and care systems to future proof health and care services for patients? Thinking about the pandemic responses, technology advancements, changes in demographics, attitudes/behavioral changes, international turmoil, climate change - What is the leadership required? What are the impacts on the workforce? How can we achieve universal access to health services?

The Future of Health Professional Education - What are the challenges, opportunities and unknowns that need to be considered by education systems to future proof student learning for evidence-based practice - thinking about the pandemic response, technology advancements, changes in demographics, attitudes/ behavioral changes, international turmoil

What leadership is required? What are the impacts on the workforce? How can we achieve universal access to ongoing learning?

\section{CONCLUSION}

In the current environment, there is a priority for professionals who can respond to the demands of practice in complex and dynamic contexts of care. Educational and workplace learning outcomes (abilities including critical thinking) therefore need to be expressed as they are applied and used in actual practice. The education experience must go beyond providing acquisition of knowledge and skills to the 'application of that knowledge and skills in the context of practice'. The competing tensions in beliefs and values seen in responses to the disruptions caused by the COVID19 pandemic highlighted the complexities of the real world. This paper reinforces the authors' belief that PBL philosophy and methodologies are appropriate for educating health professionals in the concept of integrated care because PBL requires the use of a conceptual framework of professional education that aligns with that of integrated care.

\section{REFERENCES}

Aristovnik, A. Keržič, D. Ravšelj, D. Tomaževič, N. \& Umek, L. (2020). Impacts of the COVID-19 Pandemic on Life of Higher Education Students: a Global Perspective https://www.researchgate.net/publication/343555357_Impacts_of_the COVID-19_Pandemic_on_Life_of_Higher_Education_Students_A_Global_Perspective.

Australian Government, Department of Health. (2020). Does Primary Health Care Lead to Better Health? https://wwwl.health. gov.au/internet/publications/publishing.nsf/Content/healthoatsih-pubs-linkphc $\sim$ health-oatsih-pubs-linkphc-systems $\sim$ health-oatsih-pubs-linkphc-systems3.

News, The Lancet Vol 21, June 2020, COVID-19: consequences for higher education https://www.thelancet.com/pdfs/journals/lanonc/PIIS1470-2045(20)30287-4.pdf.

Conway, J., \& Little, P. (2000). From practice to theory: Reconceptualising curriculum development for PBL. Paper presented at the 2nd Asia Pacific Conference on problem-based learning, Singapore.

Conway, J. F., Little, P., \& Fitzgerald, M. (2011). Determining frameworks for inter-professional education and core competency through collaborative consultancy: the CARE experience. Contemporary Nurse, 38(1-2), 160-170.

Deloitte Center for Higher Education Excellence. (2020). COVID-19's impact on higher education: Strategies for tackling the financial challenges facing colleges and universities https:// www2.deloitte.com/content/dam/Deloitte/us/Documents/public-sector/us-gps-covid-19-impact-on-higher-education.pdf.

Hendry, A. (2017). International Foundation for Integrated Care (IFIC) Presentation to the NSW Health - Central Coast Local Health District Symposium, February.

International Foundation for Integrated Care (IFIC). https:// www.ncbi.nlm.nih.gov/pubmed/23593072.

National Health. England: National Voices (2012) http://www. nationalvoices.org.uk/pages/narrative-person-centred-care $\% \mathrm{C} 2 \% \mathrm{~A} 0$.

New South Wales Health. (2016): Central Coast Local Health District: Report on the Pilot Study on Integrated Care, Gosford, New South Wales, Australia.

Stein, V. (2016). Integrated Care Around the World: Moving towards population health management. International Foundation for integrated care. A presentation to the NSW Central Coast Health Local Health District (NSW CCLHD - November).

World Health Organization (WHO). (2020). COVID19 Update. https://covid19.who.int/.

World Health Organization (WHO). (2016). Regional Office for 
Europe. Lessons for transforming health services delivery: compendium of initiatives in the WHO European Region. https:// www.euro.who.int/_data/assets/pdf_file/0014/303026/

Compendium. 